\section{Der Spion}

\section{zu Hause}

\section{In Kürze}

- Geräte mit digitalen Assistenten und Sprachsteuerung, wie z.B. „Smart Speaker", verbreiten sich zunehmend in Haushalten und belauschen Käuferlnnen potentiell permanent in den privatesten Räumen.

- Die Geräte sammeln personenbezogene Daten und senden diese an die Hersteller. Durch die intransparente Datenauswertung vertieft sich das Ungleichgewicht zwischen Anbietern und KonsumentInnen.

- Zukünftig könnten digitale Assistenzsysteme den Zugang zur (Online-)Welt regeln.

- Die Beurteilung dieses Geschäftsmodells wirft nicht nur datenschutzrechtliche, sondern auch gesellschaftspolitische Fragen auf.

\section{Worum geht es?}

Während Sprachsteuerung lange eine Nischenanwendung war, scheint sie mit Hilfe smarter Lautsprecher massentauglich zu werden. Die Stückzahlen verkaufter Smart Speaker pro Quartal haben sich weltweit seit 2016 im Jahresvergleich jeweils vervierfacht. Besonders groß sind die Umsätze der Anbieter im vierten Quartal wegen des Weihnachtsgeschäfts. Es gibt immer mehr Haushalte, in denen sich einer der populären digitalen Assistenten in einem Smart Speaker findet. Seit der Einsatz von Alexa \& Co. zu Hause ansteigt, ist auch eine vermehrte Nutzung auf Smartphones zu beobachten, obwohl sie dort schon länger zur Verfügung stünden. Kontinuierlich werden die Einsatzbereiche dieser Assistenzsysteme erweitert: Inzwischen werden Smart Speaker bereits bei Behörden und in manchen Hotelzimmern aufgestellt. Auch Autos haben Sprachassistenten integriert.

Alexa, Google Assistant, Siri, Cortana oder Bixby versuchen ihre NutzerInnen zu unterstützen, indem sie Fragen beantworten, auf Zuruf Nachrichten versenden, den Einkauf erledigen, Musik abspielen, Auskunft über das Wetter geben, einen Witz erzählen oder den Wecker stellen. Die meisten können mit Drittanbietersoftware um Funktionen erweitert werden, bspw. Gerätesteuerungen im Smart-Home-Bereich. Rei-bungslos funktioniert das alles noch nicht, aber es gibt auf allen Seiten große Erwartungen für die Zukunft.

Neben diesen Aufgaben ergeben sich für bestimmte Personengruppen durch die Sprachsteuerung aber auch große Vorteile. Blinde Menschen oder solche mit Störungen der Feinmotorik profitieren sehr von der Möglichkeit der Spracheingabe. Digitale Assistenten können so die Teilhabe an der digitalen Welt ermöglichen.

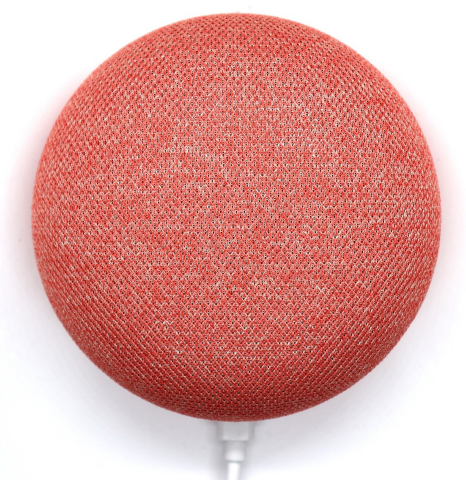

Smart Speaker lauschen ständig mit.

Damit sie das alles tun können, brauchen sie einerseits eine Verbindung zu ihren Herstellern, da die Spracherkennung in der Hersteller-Cloud erfolgt. Andererseits müssen sie permanent auf das „Zauberwort" lauschen, mit dem Sprachaufzeichnung und -auswertung aktiviert werden. In der Vergangenheit hat sich gezeigt, dass die Geräte durchaus auch Wortfetzten eines Gesprächs irrtümlich als Aktivierungsphrase interpretieren können. Durch beabsichtigte und unbeabsichtigte Aufzeichnungen entstehen sehr genaue Profile jedes Haushalts: wer und wie viele da wohnen, wann sie zu Hause sind, worüber gesprochen wird, welche Interessen die Bewohnerlnnen haben, welche ansteuerbaren Geräte verfügbar sind und vieles mehr. Durch die Möglichkeit der Verknüpfung mit Metadaten (Ort und Zeit der Anfrage, letzte Anfrage usw.) und Daten aus anderen Quellen, vornehmlich dem Smartphone und/oder der Bestellhistorie, entstehen auch sehr präzise individuelle Profile.

\section{Eckdaten}

Projekttitel: Digitale Assistenten

Projektteam: Schaber, F, Krieger-Lamina, J.,

Peissl, W.

Auftraggeber: Bundesarbeitskammer 


\section{Wesentliche Ergebnisse}

Auch wenn es Unterschiede zwischen den Anbietern gibt, kann man derzeit bei keinem von einem wirklich datenschutzfreundlichen Produkt sprechen. Alle Daten werden zentral verarbeitet, gespeichert und ausgewertet. Meist werden die Daten nur sehr spät oder überhaupt nicht gelöscht.

Für die Zukunft erwarten sich die Anbieter das große Geschäft: Conversational Commerce, sprachbasierter Einkauf, soll einen Umbruch wie bei der Einführung des Online-Handels auslösen. Über die Stimme wird nicht nur überall das Individuum identifiziert, sondern auch dessen Gemütszustand und Gesundheit erkannt. Passende Produkte sind dann nur noch einen Zuruf entfernt.

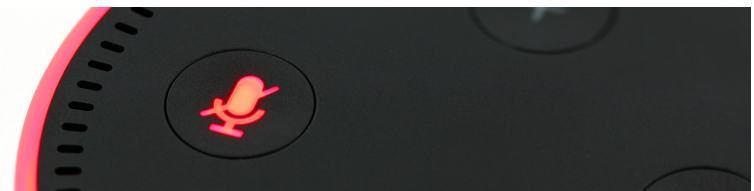

Datenauswertung verschafft den Anbietern große Vorteile

Die Auswertung der Daten verschafft den Anbietern auch auf einem anderen Gebiet einen Vorteil: Je mehr (Trainings-) Daten für ihre Algorithmen zur Verfügung stehen, desto besser werden die Systeme, desto mehr NutzerInnen werden dadurch angezogen, desto mehr Drittanbieter werden an der Plattform andocken wollen, was wiederum mehr NutzerInnen überzeugt usw. Diese Netzwerkeffekte werden bei fehlendem Gegensteuern dazu führen, dass die großen Anbieter weiter wachsen und die Konkurrenz vom Markt verdrängt wird.

Viele wollen schon jetzt mit dabei sein, um sich einen Teil des künftigen Marktes zu sichern. Wenn man in Zukunft von vernetzten Gegenständen umgeben sein wird und digitale Assistenten ständig verfügbar sowie über viele verschiedene Geräte ansprechbar sein werden, ist es wahrscheinlich, dass viele Aufgaben und Anfragen über sie abgewickelt werden. Sie werden wissen, wann wir mit wem kommunizieren, wo wir unsere Tage verbringen, was uns interessiert usw. Sie werden buchstäblich alles von uns wissen. Auf der anderen Seite könnten sie im kommerziellen Interesse ihrer Hersteller antworten und Vorschläge machen. Für NutzerInnen wird es schwierig bis unmöglich sein zu erkennen, ob und in welcher Art sie manipuliert werden. Ähnlich, wie es sich auch bei den Ergebnissen von Suchmaschinen zeigt, werden wir an erster Stelle Inhalte präsentiert bekommen, die für den Anbieter besonders profitabel sind. Jeder Assistent wird ein anderes „Ökosystem“ bedienen, andere Versandhändler, Transportunternehmen oder Urlaube vorschlagen. Die Assistenzsysteme säßen an der Schnittstelle zwischen VerbraucherInnen, Werbetreibenden, Händlern und Produzenten. Sie an dieser Machtposition zu kontrollieren, wäre schwierig.

\section{Was tun?}

\begin{abstract}
Sprachsteuerung und digitale Assistenten werden sich aller Wahrscheinlichkeit nach am Markt durchsetzen. Derzeit profitieren davon hauptsächlich die Anbieter der Assistenzsysteme, weniger deren Kundlnnen. Das Sammeln und Auswerten personenbezogener Daten sollte auch, aber nicht nur aus dem Blickwinkel des Datenschutzes beachtet werden.
\end{abstract}

- Der Schutz der Privatsphäre und anderer Grundrechte ist in einer liberal-demokratischen Gesellschaft unabdingbar. Der massenhafte Einsatz dieser Geräte gefährdet die Privatsphäre und damit die Eckpfeiler der Demokratie. Die Politik sollte sich daher genau ansehen, welche Möglichkeiten der Regulierung bestehen, welche Ressourcen dafür benötigt werden sowie welches Wissen dafür vielleicht noch fehlt und erforscht werden muss.

- Anbieter dieser Systeme könnten Daten auch lokal auf den Geräten verarbeiten, nur aggregierte Daten sammeln bzw. auswerten und sich grundsätzlich überlegen, wie sie ihr Geschäft durch den Wettbewerbsvorteil der privatsphären-freundlichen Produktgestaltung verbessern und die Erfüllung bestehender Normen dadurch genauer einhalten können.

- KonsumentInnen sollten sich genau ansehen, ob digitale Assistenten das erfüllen, was sie sich erwarten, und ob der Nutzen gegenüber den dargestellten Nachteilen einen Einsatz rechtfertigt. Falls diese Überlegungen zum Kauf so eines Geräts führen, dann sollte der relativ beste Schutz der Privatsphäre den Ausschlag bei der Kaufentscheidung geben. Damit könnte auf lange Sicht eine Motivation für Hersteller entstehen, das Konzept Privacy-byDesign besser umzusetzen.

\section{Zum Weiterlesen}

Schaber, F., Krieger-Lamina, J., Peissl, W. (2019): Digitale Assistenten, Projektbericht 01-2019, ITA: Wien epub.oeaw.ac.at/ita/ita-projektberichte/2019-01.pdf

\section{Kontakt}

Jaro Krieger-Lamina

E-mail: tamail@oeaw.ac.at

Telefon: +43(1)51581-6582

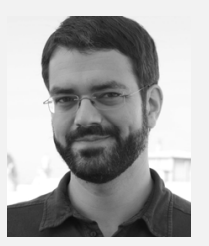

IMPRESSUM: Medieninhaber: Österreichische Akademie der Wissenschaften; Juristische Person öffentlichen Rechts (BGBI. 569/1921 i.d.F. BGBI. I 31/2018); Dr. Ignaz Seipel-Platz 2 A-1010 Wien; Herausgeber: Institut für Technikfolgen-Abschätzung (ITA); Apostelgasse 23, A-1030 Wien; www . oeaw ac at/ita | Erscheinungsweise: Die ITA-Dossiers erscheinen unregelmäßig und dienen der Veröffentlichung der Forschungsergebnisse des ITA. Die Dossiers werden in kleiner Auflage gedruckt und open access über das Portal „epub.oeaw“ zur Verfügung gestellt: epub. oeaw.ac.atita/ita-dossiers | ISSN: 2306-1960 Dieses Werk ist lizenziert unter einer Creative Commons Namensnennung 4.0 International Lizenz: creativecommons. ITA-Dossier Nr. 44, Juli 2019, epub. oeaw ac. at/ita/ita-dossiers/ita-dossier044. pdf | Koautoren: Schaber, F., Peissl, W. 